\title{
Fluorescence Imaging of Underexpanded Jets and Comparison with CFD
}

\author{
J.A. Wilkes*, C.E. Glass ${ }^{\dagger}$, P.M. Danehy ${ }^{\ddagger}$, R.J. Nowak ${ }^{\S}$ \\ NASA Langley Research Center, Hampton VA, 23681-2199
}

\begin{abstract}
An experimental study of underexpanded and highly underexpanded axisymmetric nitrogen free jets seeded with $0.5 \%$ nitric oxide (NO) and issuing from a sonic orifice was conducted at NASA Langley Research Center. Reynolds numbers based on nozzle exit conditions ranged from 770 to 35,700 , and nozzle exit-to-ambient jet pressure ratios ranged from 2 to 35. These flows were non-intrusively visualized with a spatial resolution of approximately $0.14 \mathrm{~mm} \times 0.14 \mathrm{~mm} \times 1 \mathrm{~mm}$ thick and a temporal resolution of $1 \mu \mathrm{s}$ using planar laser-induced fluorescence (PLIF) of NO, with the laser tuned to the stronglyfluorescing UV absorption bands of the $Q_{1}$ band head near $226.256 \mathrm{~nm}$. Three laminar cases were selected for comparison with computational fluid dynamics (CFD). The cases were run using GASP (General Aerodynamic Simulation Program) Version 4. Comparisons of the fundamental wavelength of the jet flow showed good agreement between CFD and experiment for all three test cases, while comparisons of Mach disk location and Mach disk diameter showed good agreement at lower jet pressure ratios, with a tendency to slightly underpredict these parameters with increasing jet pressure ratio.
\end{abstract}

\section{Nomenclature}

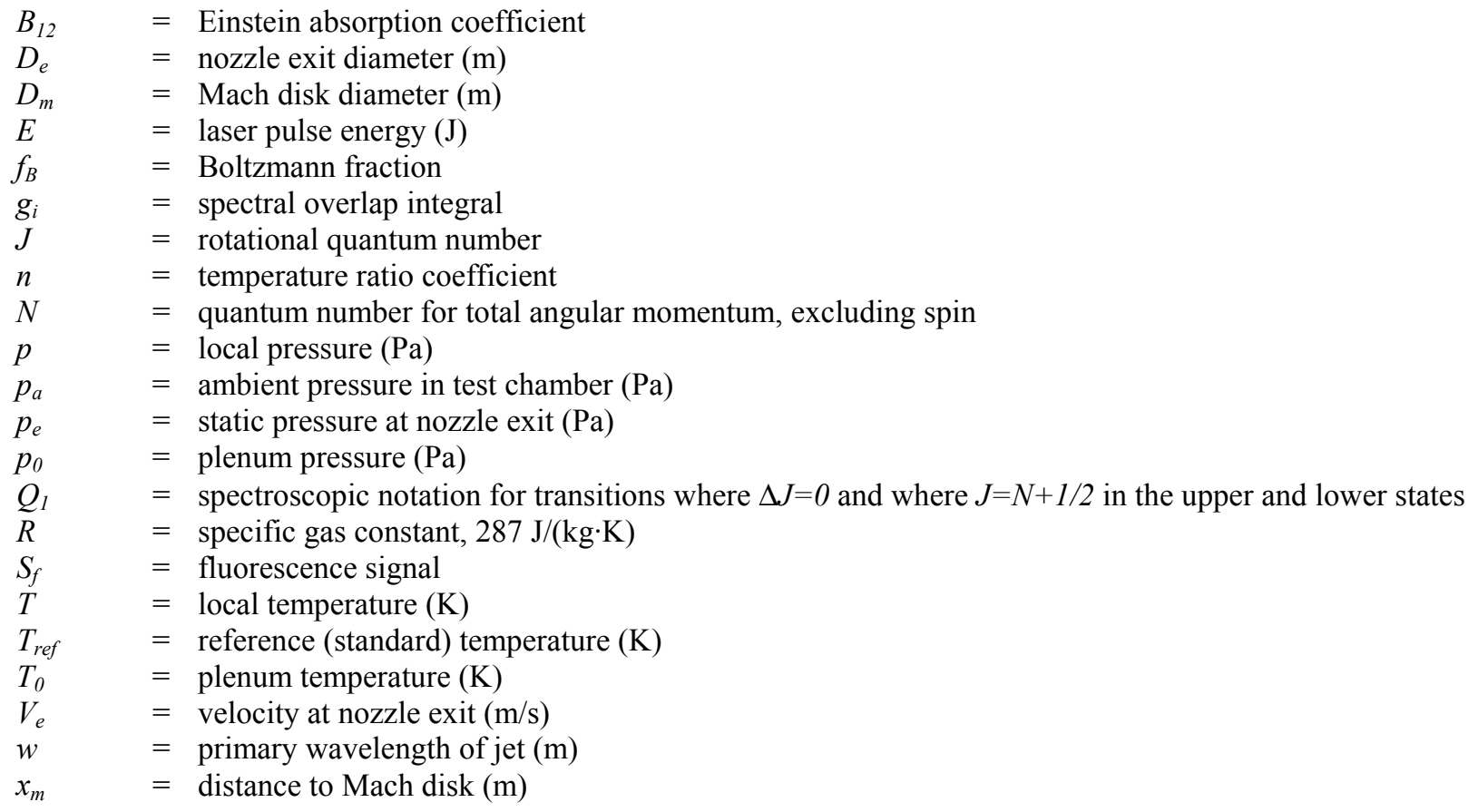

\footnotetext{
* PhD Student, Department of Physics, The College of William and Mary, Williamsburg,VA and NASA Graduate CoOp Student, Advanced Sensing and Optical Measurement Branch, MS 493, AIAA Student Member.

${ }^{\dagger}$ Research Scientist, Aerothermodynamics Branch, MS 408A, AIAA Senior Member.

${ }^{*}$ Research Scientist, Advanced Sensing and Optical Measurement Branch, MS 493, AIAA Associate Fellow.

${ }^{\S}$ Research Scientist, Aerothermodynamics Branch, MS 408A, AIAA Member 


$\begin{array}{ll}Y_{N O} & =\text { mass fraction of nitric oxide } \\ \gamma & =\text { ratio of specific heats } \\ \mu_{e} & =\text { dynamic viscosity at nozzle exit }(\mathrm{Pa} \cdot \mathrm{s}) \\ \mu_{r e f} & =\text { reference }(\text { standard }) \text { dynamic viscosity }(\mathrm{Pa} \cdot \mathrm{s}) \\ \rho & =\text { gas density }\left(\mathrm{kg} / \mathrm{m}^{3}\right) \\ \rho_{e} & =\text { gas density at nozzle exit }\left(\mathrm{kg} / \mathrm{m}^{3}\right) \\ \Phi & =\text { fluorescence yield } \\ \chi_{N 2} & =\text { mole fraction of nitrogen } \\ \chi_{N O} & =\text { mole fraction of nitric oxide }\end{array}$

\section{Introduction}

$\mathrm{H}$ IGHLY underexpanded axisymmetric free jets are important flows of interest, for both fundamental fluid mechanics studies and for practical applications. Such jets have been investigated extensively in the study of propulsive jets, rocket exhaust plumes, and vehicle control jets. ${ }^{1-7}$ The present study was motivated by the NASA Shuttle Return to Flight (RTF) investigation into the effects of flow through vehicle wing breaches. Computational fluid dynamics (CFD) represents a vital tool for evaluating flight damage scenarios since no set of ground-based experiments could anticipate every potential vehicle damage scenario nor entirely replicate the high-energy flight environment. When applying computational tools to engineering applications, understanding the accuracy and limitations of that tool is obviously of critical importance. It was therefore desired to compare computations to experimental results. To that end, the present experimental work was designed to generate flows at conditions comparable to those expected to be encountered in flight, but with relatively simple geometries and well-defined boundary conditions.

The flow environments encountered in these tests include regions of low static pressure, turbulent and/or threedimensional flow structures, and regions with both strong and weak density gradients. Such conditions, though frequently encountered in aerospace simulation facilities, cannot be satisfactorily visualized using traditional pathaveraged techniques such as schlieren and shadowgraph. An alternative approach was therefore required. For this study, we have used PLIF of nitric oxide (NO) to image underexpanded jets issuing into a low-pressure chamber through a converging nozzle. The resulting flow visualization images allowed the size and location of major flow features to be compared with the results of CFD simulations, as well as with previous experimental and computational results.

\section{Experimental Equipment and Procedures}

\section{A. Test Apparatus}

Tests were conducted at NASA Langley Research Center using the test section of the 15-Inch Mach 6 Wind Tunnel as a vacuum chamber. The toxic gas bottles were stored in a ventilated, alarmed toxic-gas cabinet. Nitrogen $\left(\mathrm{N}_{2}\right)$ was seeded with $0.5 \%$ nitric oxide $(\mathrm{NO})$. The gas mixture was introduced into a stainless steel plenum where it was convectively heated to approximately $500 \mathrm{~K}$ by the surrounding metal. The gas was heated to attain flows with Reynolds numbers lower than could be achieved with unheated gas. The steel of the plenum was itself heated by electrical heat tape wrapped around the exterior of the model and covered with insulation. Due to the large mass of the model, preheat times of approximately four hours were required and fine-tuning of the gas temperature was not feasible. After passing through the plenum, the heated gas exhausted through a converging sonic nozzle and into the vacuum chamber. The nozzle geometry is illustrated in Fig. 1. (This figure also shows the grid used in the CFD computations, described in greater detail in the section below on Computational

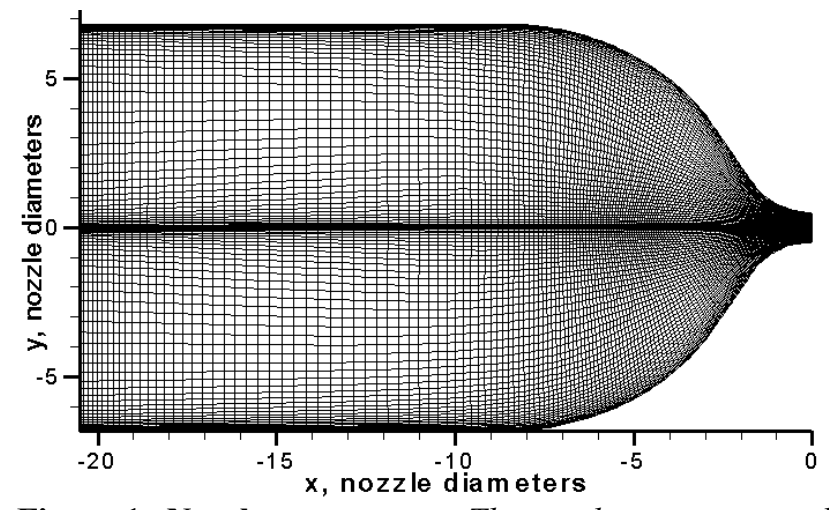

Figure 1. Nozzle geometry. The nozzle geometry used in these tests is illustrated above, showing the grid used in the CFD computations. While the CFD computations were run axially symmetric, the nozzle grid geometry is shown here mirrored over the horizontal axis for illustrative purposes. 
Methodology.) Mass flow controllers adjusted the flow rates, which determined the plenum pressure and nozzle exit Reynolds number. A schematic including these elements of the test apparatus is shown in Fig. 2. A thermocouple under the insulation and against the model surface measured its temperature. Two thermocouples and one pressure transducer were inserted through feed-through holes in the model. They measured the gas temperature, $T_{0}$, and pressure, $p_{0}$, in the plenum, upstream of the converging nozzle. Measuring these quantities made it possible to calculate conditions at the nozzle exit, and therefore the exit Reynolds number, as described in detail in the next section.

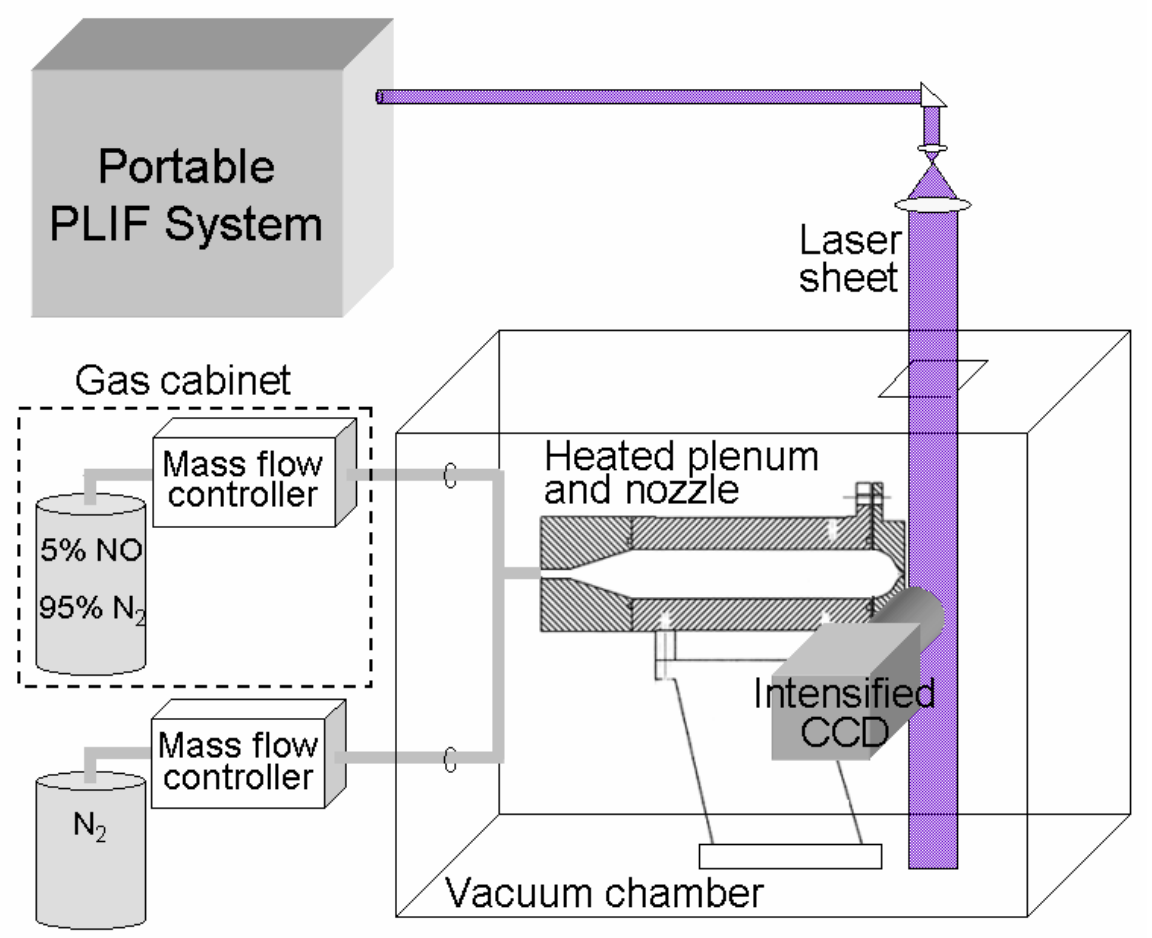

Figure 2. Schematic of experimental setup. Gas is plumbed through a heated plenum and nozzle into a vacuum chamber. A laser sheet enters the top of the vacuum chamber and excites nitric oxide molecules in the flow. An intensified $C C D$ camera positioned at right angles to the laser sheet images the fluorescence.

\section{B. Test Parameters}

Two parameters were independently varied: Reynolds number $\left(R e_{e x i t}\right)$ and jet pressure ratio $(J P R)$. $R e_{\text {exit }}$ was defined in terms of the diameter $D_{e}(\mathrm{~m})$, density $\rho_{e}\left(\mathrm{~kg} / \mathrm{m}^{3}\right)$, velocity $V_{e}(\mathrm{~m} / \mathrm{s})$, and dynamic viscosity $\mu_{e}(\mathrm{~Pa} \cdot \mathrm{s})$ at the nozzle exit, as given by Eq. (1).

$$
\operatorname{Re}_{\text {exit }}=\frac{\rho_{e} V_{e} D_{e}}{\mu_{e}}
$$

The nozzle used in these tests had an exit diameter of $D_{e}=2.4 \mathrm{~mm}\left(0.095 \mathrm{in}\right.$.). $R e_{\text {exit }}$ was varied by changing the mass flow rates and gas temperature, and was calculated based on measured plenum conditions, as described below. $J P R$ was defined as the ratio of the static pressure at the nozzle exit, $p_{e}$, to the ambient pressure in the test chamber, $p_{a}$, according to Eq. (2), and was varied by changing the test chamber pressure.

$$
J P R=\frac{p_{e}}{p_{a}}
$$


For a given $R e_{\text {exit }}$, the maximum attainable $J P R$ was limited by the minimum attainable pressure in the vacuum chamber, which was typically about $130 \mathrm{~Pa}$ (1 Torr, $0.02 \mathrm{psi})$.

Nozzle exit temperature $T_{e}(\mathrm{~K})$ and velocity $V_{e}(\mathrm{~m} / \mathrm{s})$ were calculated from the measured quantities $T_{0}$, and pressure, $p_{0}$, assuming inviscid adiabatic flow at the nozzle exit, according to Eqs. (3) and (4): ${ }^{8}$

$$
\begin{gathered}
T_{e}=\frac{T_{0}}{1+\left(\frac{\gamma-1}{2}\right)} \\
V_{e}=\sqrt{\gamma R T_{e}},
\end{gathered}
$$

where $\gamma$ is the ratio of specific heats of the gas ( $\gamma=1.4$ for nitrogen), and $R$ is the specific gas constant with a value of $287 \mathrm{~J} /(\mathrm{kg} \cdot \mathrm{K})$. Furthermore, with the added assumption of isentropic flow, nozzle exit pressure $p_{e}(\mathrm{~Pa})$ and density $\rho_{e}\left(\mathrm{~kg} / \mathrm{m}^{3}\right)$ can be calculated using Eqs. (5) and (6): ${ }^{8}$

$$
\begin{gathered}
p_{e}=\frac{p_{0}}{\left(1+\frac{\gamma-1}{2}\right)^{\gamma /(\gamma-1)}} \\
\rho_{e}=\frac{p_{0}}{R T_{0}\left(1+\frac{\gamma-1}{2}\right)^{1 /(\gamma-1)}} .
\end{gathered}
$$

The dynamic viscosity $\mu_{e}(\mathrm{~Pa} \cdot \mathrm{s})$ at the nozzle exit was calculated using the power law approximation given in Eq. (7):

$$
\mu_{e}=\mu_{r e f}\left(\frac{T_{e}}{T_{r e f}}\right)^{n} .
$$

This power law accounts for the effect of temperature on dynamic viscosity while neglecting the slight dependence on pressure. Reference 9 gives an empirical value of $n=0.67$ for nitrogen, where $T_{\text {ref }}=273 \mathrm{~K}\left(32{ }^{\circ} \mathrm{F}\right)$ and $\mu_{\text {ref }}=$ $1.663 \times 10^{-5} \mathrm{~Pa} \cdot \mathrm{s}$. Values for nitrogen were used because the gas consisted of $99.5 \% \mathrm{~N}_{2}$, and because NO, which made up the remaining $0.5 \%$, has a viscosity similar to that of $\mathrm{N}_{2} .{ }^{9}$

\section{Laser System}

The laser system consists of three main components: a pump laser, a pulsed dye laser (PDL), and a wavelength extender (WEX). An injection-seeded Nd:YAG laser operating at $10 \mathrm{~Hz}$ pumps a tunable PDL. The output of the dye laser and the residual infrared from the Nd:YAG are combined in a WEX containing both a doubling and a mixing crystal. The resulting output is tuned to a wavelength of $226.256 \mathrm{~nm}$, chosen to excite the strongly fluorescing spectral lines of $\mathrm{NO}$ near the $Q_{l}$ branch head. (In this notation, $Q$ indicates that the rotational quantum number $J$ is the same for both the upper and lower states of the transition; i.e., $\Delta J=0$. The subscript 1 indicates that, in both the upper and lower states involved in the transition, $J$ is related to $N$ by $J=N+1 / 2$, where $N$ is the quantum number for total angular momentum, excluding spin.)

A monitoring gas cell system is used to ensure that the laser is tuned to the correct spectral line of NO. The gas cell contains a low-pressure mixture of $5 \% \mathrm{NO}$ in $\mathrm{N}_{2}$. A quartz window serves as a beam splitter and sends a small portion of the laser energy through windows on either side of the gas cell. A photomultiplier tube (PMT) monitors the fluorescence intensity through a third window at right angles to the path of the laser beam. The components of this laser system are mounted within a two-level, enclosable, portable cart. A photograph of this portable PLIF cart 
is shown in Fig. 3 with all the panels removed to show the internal components. When all of the panels are in place, a single monochromatic ultraviolet laser beam exits the cart, creating a relatively safe operating environment.

\section{PLIF Flow Visualization}

Optics form the beam into a $100 \mathrm{~mm}$ wide $\mathrm{x} \sim 1 \mathrm{~mm}$ thick laser sheet, which is directed vertically downward through a window in the top of the test section. The scaffolding upon which the optics are mounted and a translation stage allow the laser sheet to be precisely aligned with the center of the nozzle exit. The laser sheet excites NO molecules in the flow. Fluorescence is imaged onto a gated, intensified CCD at a viewing angle normal to the laser sheet. The schematic of the experimental setup shown in Fig. 1 includes these elements. Additionally, a 1$\mathrm{mm}$ thick Schott glass UG5 filter was placed in front of the camera lens in order to attenuate scattered light at the laser's frequency, which is monochromatic, while transmitting the majority of the comparatively broadband fluorescence.

Before every run, mass flow rates were established using the mass flow controllers. The total mass flow rate determined the plenum pressure, which in turn determined the desired exit Reynolds number. The test chamber was brought to the pressure necessary to achieve the desired jet pressure ratio using a steam ejector and vacuum pumps. The intensified CCD was coordinated so that the start of data acquisition coincided with the firing of the laser. Sets of 100 single-shot images were acquired for a range of $R e_{\text {exit }}$ and $J P R$.

The absolute intensity at any given pixel in a PLIF image has a functional dependence on many local flow

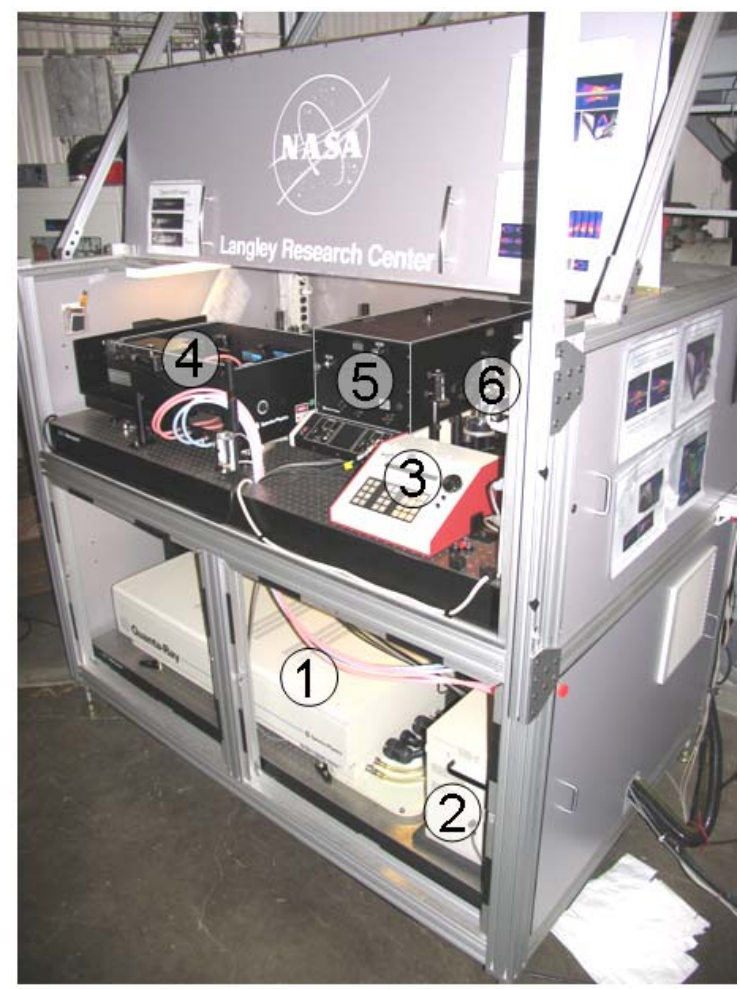

Figure 3. The portable PLIF system. Here, the system is shown with panels removed. Components include: (1) Nd:YAG laser; (2) dye circulators with Rhodamine 590 and Rhodamine 610 laser dyes in a methanol solvent; (3) wavelength controller for the (4) pulsed dye laser; (5) wavelength extender; and (6) low pressure monitoring gas cell. parameters, including temperature, pressure, and species mole fractions. A simplified expression for fluorescence signal is given below in Eq. (8), where $S_{f}$ is the fluorescence signal; $E$ is the energy in a single laser pulse; $\rho$ is the local gas density; $\Phi$ is the fluorescence yield; $\chi_{\mathrm{NO}}$ is the local mole fraction of NO; $f_{B}$ is the Boltzmann fraction; $B_{12}$ is the Einstein absorption coefficient for the probed transition; and $g_{i}$ is the spectral overlap integral. The quantities $\Phi, f_{B}$ and $g_{i}$ are functions of the local temperature; $\Phi$ and $g_{i}$ also depend on the local pressure.

$$
S_{f} \propto E \rho \Phi \chi_{N O}\left[\sum f_{B} B_{12} g_{i}\right]
$$

Since the fluorescence intensity depends on so many local flow parameters, variations in any one of these parameters will affect the resulting intensity in a PLIF image. While this means that the interpretation of PLIF images is not always straightforward, it also means that PLIF images are well suited to detecting fluctuations within a flowfield. We have previously reported using the flow visualization images from this study, along with a customized data-processing procedure, to accentuate local fluctuations in fluorescence intensity. This information led to the identification of a correlation between flow parameters and the downstream distance at which these flows begin to exhibit unsteady behavior. ${ }^{10}$

Data acquisition of measured flow parameters was initiated by the tunnel operator; included in these measured parameters was a hand-pressed trigger, indicating the beginning and end of image acquisition. Images were acquired at the laser repetition rate of $10 \mathrm{~Hz}$ with a $1 \mu \mathrm{s}$ camera gate. A camera lens imaged a region of the flow through an image intensifier onto a 512 pixel x 512 pixel CCD array (Princeton Instruments PI-MAX II). Most images were acquired using a $105 \mathrm{~mm}$ UV Nikkor lens, resulting in a spatial resolution of approximately 7 pixels $/ \mathrm{mm}$. For select cases, where a larger field of view was desired, an $45 \mathrm{~mm}$ focal length UV Cerco lens was used instead, resulting in a spatial resolution of approximately 3 pixels $/ \mathrm{mm}$. This temporal and spatial resolution 
was more than sufficient to resolve many flow structures of interest. Further details of the laser and camera system can be found in Ref. 11.

\section{Data Analysis and Computational Methodology}

\section{A. Image Processing and Analysis}

As stated above, sets of 100 single-shot images were acquired for a range of unit $R e_{\text {exit }}(177$ to 35,700$)$ and $J P R$ (1.8 to 38). Background images were also acquired on each day of testing for a range of vacuum chamber pressures. During the acquisition of these background images, the laser was fired but no gas was flowing through the nozzle. Any nonzero intensity in these background images is attributed to either camera dark current or the laser scatter and room light not blocked by the filter in front of the camera lens. Background images were created from the average of 100 single-shot images in order to smooth out random shot-to-shot variations in background intensity.

Single-shot images were processed to correct for background scattered light and camera dark current as well as spatial variations in laser sheet intensity. Both the background image and the single-shot images were smoothed with a filter (a 3 pixels x 3 pixels rotationally symmetric low-pass Gaussian filter with a standard deviation of 1) prior to additional processing in order to reduce noise in the images. A background image was then subtracted from the filtered single-shot image. Images were subsequently corrected for laser nonuniformities. Conveniently, jet gas containing nitric oxide diffused relatively uniformly into the test chamber in regions away from the jet. The fluorescence from the diffuse nitric oxide in these regions provided a convenient laser-energy reference, allowing the spatial variation in the laser intensity to be corrected. This was accomplished by first selecting an area of the image above the core of the jet flow and then establishing the average pixel intensity along each column in that region. (Note that raw images were 512 x 512 pixels; images presented in this paper have been cropped top and bottom to show the regions of greatest interest.) Finally, the background-corrected images were divided by this laser sheet profile. Since the laser sheet is typically brightest in the center of the sheet, the result of dividing an image by the laser sheet profile is to increase the brightness of the left and right edges of the image relative to the central part of the image.

\section{B. Selection of Test Cases}

Three test cases were selected for the purposes of comparison with CFD. Three different jet pressure ratios were selected, and for ease of comparison with CFD, all three selected cases were laminar. Velocimetry data were taken at each of these three test conditions, although they are not reported herein as the results are still being analyzed. Measured parameters recorded by the data acquisition system during these velocimetry runs was processed and served as the input conditions to the CFD code, as described below. Flow visualization images were acquired at conditions similar to these three cases. Due to hardware limitations, the gas temperature was only coarsely controllable (within about $50 \mathrm{~K}$ ), and the test chamber static pressure was only repeatable within about 0.1 Torr $(0.002 \mathrm{psi}$, $13 \mathrm{~Pa}$ ). These variations led to slight variations between the

\begin{tabular}{|c|c|c|c|c|c|c|c|c|}
\hline \multirow{2}{*}{$\begin{array}{l}\text { Test } \\
\text { Case }\end{array}$} & \multirow[t]{2}{*}{$J P R$} & \multirow[t]{2}{*}{$R e_{e x i t}$} & $T_{0}$ & \multicolumn{2}{|c|}{$p_{0}$} & \multicolumn{3}{|c|}{$p_{a}$} \\
\hline & & & $K$ & $P a$ & $p s i$ & $P a$ & $p s i$ & Torr \\
\hline$A$ & $\begin{array}{r}4.2 \\
(3.8)\end{array}$ & $\begin{array}{r}835 \\
(846)\end{array}$ & $\begin{array}{r}588 \\
(544)\end{array}$ & $\begin{array}{r}5090 \\
(4710)\end{array}$ & $\begin{array}{r}0.738 \\
(0.683)\end{array}$ & $\begin{array}{r}646 \\
(657)\end{array}$ & $\begin{array}{r}0.094 \\
(0.095)\end{array}$ & $\begin{array}{r}4.84 \\
(4.93)\end{array}$ \\
\hline$B$ & $\begin{array}{r}18.2 \\
(16.8)\end{array}$ & $\begin{array}{r}1101 \\
(1095)\end{array}$ & $\begin{array}{r}605 \\
(559)\end{array}$ & $\begin{array}{r}6941 \\
(6290)\end{array}$ & $\begin{array}{r}1.01 \\
(0.91)\end{array}$ & $\begin{array}{r}201 \\
(198)\end{array}$ & $\begin{array}{r}0.029 \\
(0.029)\end{array}$ & $\begin{array}{r}1.51 \\
(1.49)\end{array}$ \\
\hline$C$ & $\begin{array}{r}31.4 \\
(27.6)\end{array}$ & $\begin{array}{r}1230 \\
(1136)\end{array}$ & $\begin{array}{r}580 \\
(590)\end{array}$ & $\begin{array}{r}7379 \\
(6950) \\
\end{array}$ & $\begin{array}{r}1.07 \\
(1.01)\end{array}$ & $\begin{array}{r}124 \\
(133)\end{array}$ & $\begin{array}{r}0.018 \\
(0.019)\end{array}$ & $\begin{array}{r}0.93 \\
(1.00)\end{array}$ \\
\hline
\end{tabular}

Table 1. Test conditions. Jet pressure ratio, exit Reynolds number, plenum temperature, plenum pressure, and test chamber pressure for the three CFD test cases are presented in this table. Values in parentheses are conditions for three flow visualization runs taken at conditions similar to those for which CFD was performed.

conditions at which velocimetry data were acquired (and at which CFD computations were performed) and those at which flow visualization images were acquired. Measured and calculated test conditions for the three test cases are summarized in Table 1, with data for flow visualization conditions in parentheses. 


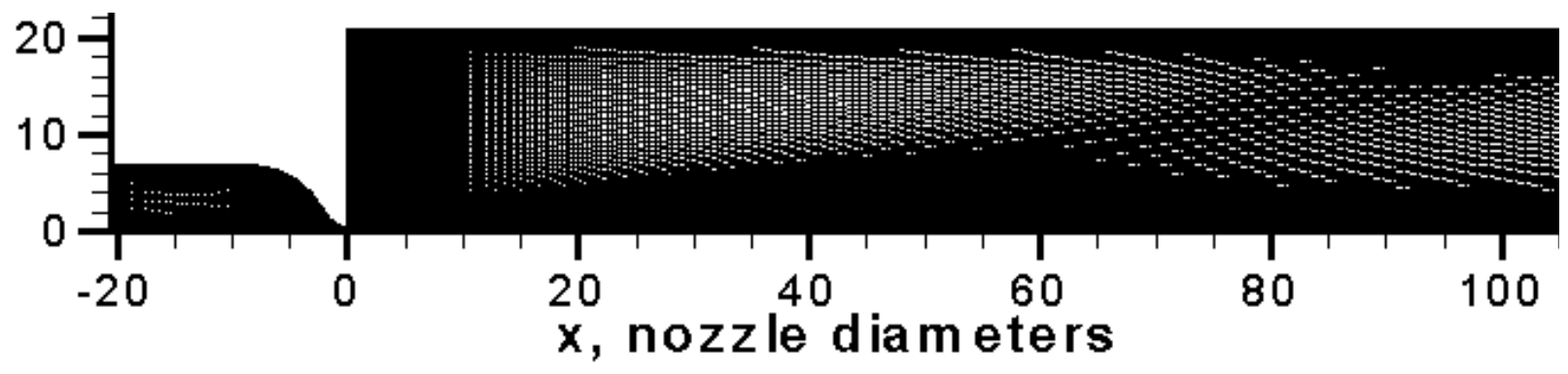

Figure 4. Grid geometry. Here, the grid geometries for zones 1 (plenum and nozzle) and 2 (free expansion region) are shown, with distances given in terms of nozzle diameters ( $D_{e}=0.095$ inches).

\section{Computational Fluid Dynamics}

The CFD for the present study was performed using the General Aerodynamic Simulation Program (GASP) Version 4 software from AeroSoft, Inc. ${ }^{12}$ Previous experience with the software for a similar study ${ }^{13}$ showed that the computational and experimental results compared well; therefore, the software was considered well-suited for the present study.

The grid used to simulate the jet consisted of two zones: a subsonic plenum and nozzle zone, consisting of 148 axial by 48 radial cells, and a free expansion zone, consisting of 320 axial by 132 radial cells. The plenum and nozzle zone profile matched that of the hardware used for the experiment. To capture the free jet, a grid larger than the jet was utilized in the jet expansion portion of the grid. The grids for both zones are shown in Fig. 4. The stagnation condition for each case was applied to the subsonic zone inflow boundary. An adiabatic, no-slip wall boundary condition was applied to the subsonic zone wall and the adjacent wall of the free expansion zone. The chamber back pressure for each case was applied to the outer grid boundary of the free expansion zone. Additionally, because the grid models only a slice of the free jet, an axisymmetric boundary condition was applied to the singular axis in the axial direction.

Grid sequencing was employed to produce the steady-state solution. A coarse grid of every fourth point of the i$\mathrm{j}$ plane provided the first converged solution. Then, a converged solution was obtained on the medium grid of every other point, and finally, the solution was converged on the fine grid. The solution was assumed converged for each grid sequence when the error norm became constant and no longer decreased. For the fine grid convergence, comparisons were made between three solutions separated by 50,000 integration cycles, which showed little difference in the free jet expansion region for Cases B and C. In Case A, nonphysical, time-dependent, vortical flow features appeared beyond a downstream distance of approximately 12 nozzle diameters. These features are artifacts of the boundary conditions imposed on the outer grid boundary in the free expansion zone, and potentially could have been eliminated following the methodology outlined in Ref. 14. In that work, the authors encountered a similar problem with computations involving a jet issuing into quiescent air. To reduce these types of numerical artifacts, they imposed a slight pressure gradient across the external domain. They found that this produced an external flow with a velocity of approximately $7 \mathrm{~m} / \mathrm{s}$, but that good convergence was found in the downstream region within a reasonable number of integration cycles. ${ }^{14}$

Time integration in the present study was performed by the Gauss-Seidel technique with the $3^{\text {rd }}$ order, upwind biased, AUSM+ inviscid flux scheme. The Navier-Stokes numerical computation was simulated with thin-layer and cross-derivative terms included in the viscous flux. Laminar flow was assumed for the perfect gas mixture of $\mathrm{N}_{2}$, NO, and air. Sutherland's law for viscosity and conductivity was employed to determine the gas transport properties with a Prandl number of 0.72 , and diffusivity was modeled using a constant Schmidt number of 0.7.

In the present work, we were interested in comparing PLIF flow visualization images with computational results. This comparison focused on the near field of the jet. For comparisons of flow structures, a converged solution was needed in regions near the Mach disk, as well as regions upstream of the Mach disk; this required distance was approximately 3 nozzle diameter for Case A. Therefore, stability of the solution down to 12 nozzle diameters was considered more than sufficient for the purpose of jet structure comparisons. One additional consideration for Case A was that in the experimental flowfield, the mixing of the jet with the ambient air downstream resulted in quenching of $\mathrm{NO}$ fluorescence by oxygen. In the flow visualization image similar to Case A, significant attenuation of the PLIF signal was observed beyond about 12 nozzle diameters because of this effect. For these reasons, we have chosen to limit our presentation of Case A results to 12 nozzle diameters downstream of the nozzle exit. 


\section{Computational Flow Imaging}

As discussed in the earlier section on PLIF flow visualization, the relative intensity in a PLIF image depends on many local flow parameters. Most significant among these are the local mole fraction of NO and the local temperature and pressure. Thus, maps of computational results showing individual flow parameters (e.g. maps of Mach number, density, pressure, temperature, etc.) qualitatively bear little resemblance to PLIF images of flows at similar conditions. Others have employed a method known as computational flow imaging (CFI), which uses CFD maps of temperature, pressure, and other flow properties, as input to calculations of fluorescence signal. CFI is thus able to generate theoretical PLIF images. ${ }^{15,16}$ As a first approximation, we have employed an elementary form of the CFI methodology. This formulation approximates the Voigt profile as a Gaussian function, allowing the overlap integral to be evaluated analytically. ${ }^{17} \quad$ It assumes the laser wavelength is tuned to the center of the absorption line shape at the $Q_{l}$ band head $(226.256 \mathrm{~nm})$, that the laser has a spectral line width of $0.5 \mathrm{~cm}^{-1}$, and that the gas is composed of nitrogen (with $\chi_{N 2}$ $=99.5 \%$ ) and nitric oxide (with $\chi_{N O}=0.5 \%$ ). This last assumption is valid in the core of the jet where little mixing with the ambient gas has occurred; it breaks down along the outer edges of the jet and sufficiently far downstream, where the jet fluid has mixed with ambient air and quenching by oxygen reduces the fluorescence yield. Maps of $Y_{N O}$, the mass fraction of nitric oxide, for the three test cases are presented in Fig. 5[JAW1]. We, showing increased mixing in these regions. In our calculations, we also chose to neglect the effect of Doppler shifts on the absorption lineshape, an effect which reduces the intensity of the experimental images; this approximation is appropriate for the conditions of these tests everywhere but close to
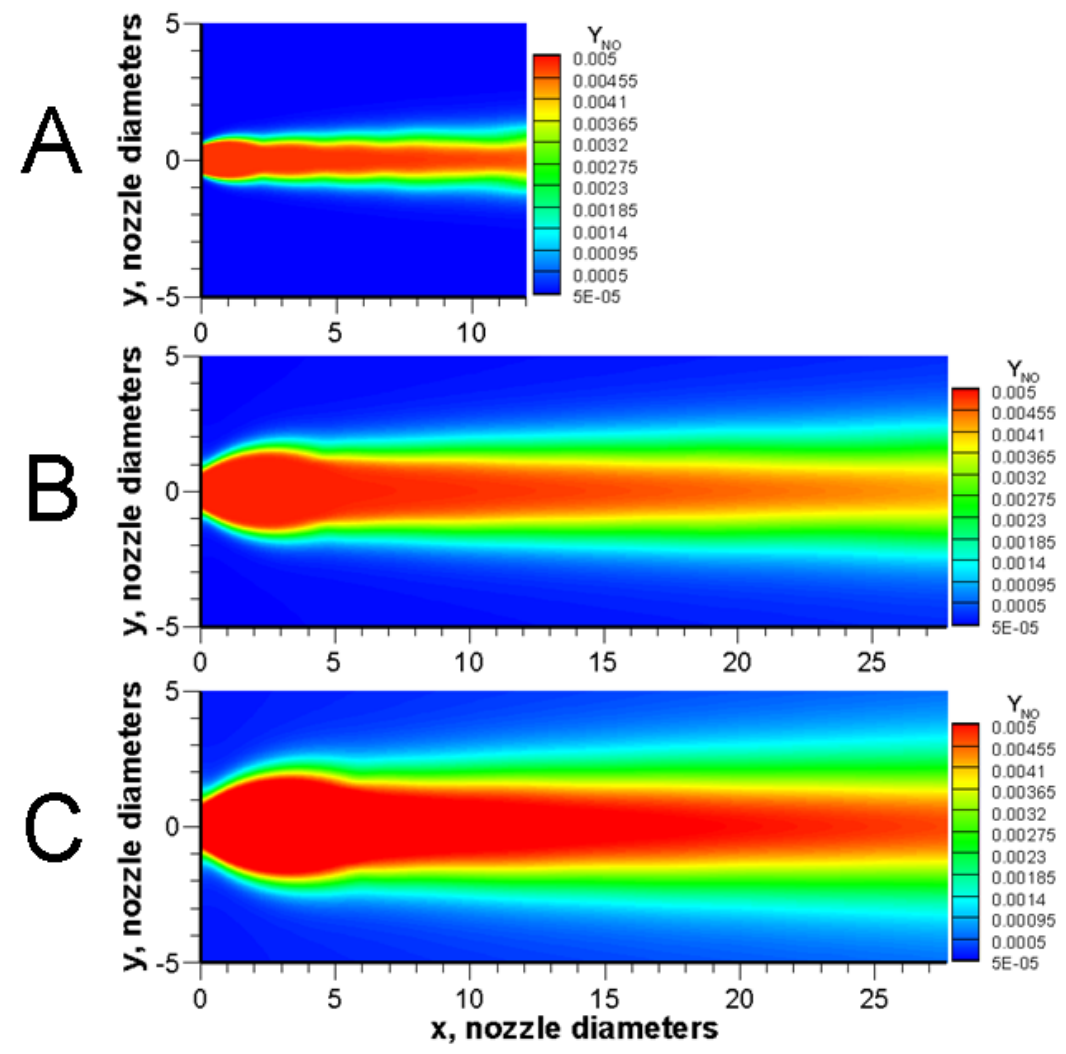

Figure 5. Nitric oxide mass fraction. Maps of the mass fraction of nitric oxide are shown for the three CFD test cases of the present study. Images have been cropped to show regions where minimal mixing has occurred in the core of the jet.

the nozzle exit, as the major component of velocity was in the axial direction, perpendicular to the laser sheet.

In this formulation, theoretical fluorescence intensity was first calculated for a range of temperatures $(100 \mathrm{~K}$ to $500 \mathrm{~K}$, the approximate range of temperatures expected in these tests) and pressures ( $2 \mathrm{~Pa}$ to $30,000 \mathrm{~Pa}$, a range somewhat greater than that expected in these tests) using the PLIF equations found in Refs. 17-22. Next, these calculated relative intensities were plotted versus pressure for five different temperatures, as shown in Fig. 6. Second-order polynomials - functions of pressure - were found to give good fits to these data points. The coefficients of these polynomials were then plotted as a function of temperature and fitted with power law fits. The result was a simplified equation for fluorescence intensity as a function of temperature, pressure, and mole fraction with semi-empirically determined coefficients which are valid for the excitation wavelength, $\mathrm{NO}$ and $\mathrm{N}_{2}$ mole fraction, and pressure and temperature ranges stated above:

$$
S_{f} \propto \chi_{N O}\left(-0.0018 T^{-2.12} p^{2}+46 T^{-1.9} p\right)
$$


Here, $S_{f}$ is the fluorescence intensity, $p$ is the pressure in pascals $\left(\mathrm{Pa}=\mathrm{N} / \mathrm{m}^{2}\right)$ and $\mathrm{T}$ is the temperature in Kelvin $(\mathrm{K})$. Images were generated in Tecplot by merging variables according to the above equation. Note that, while the PLIF equations here have been formulated in terms of the mole fractions of the constituent gas species, GASP gave species mass fractions as an output variable. Since the molecular mass of NO, $\mathrm{N}_{2}$, and air are so similar, mass fraction was simply used in place of mole fraction in these calculations.

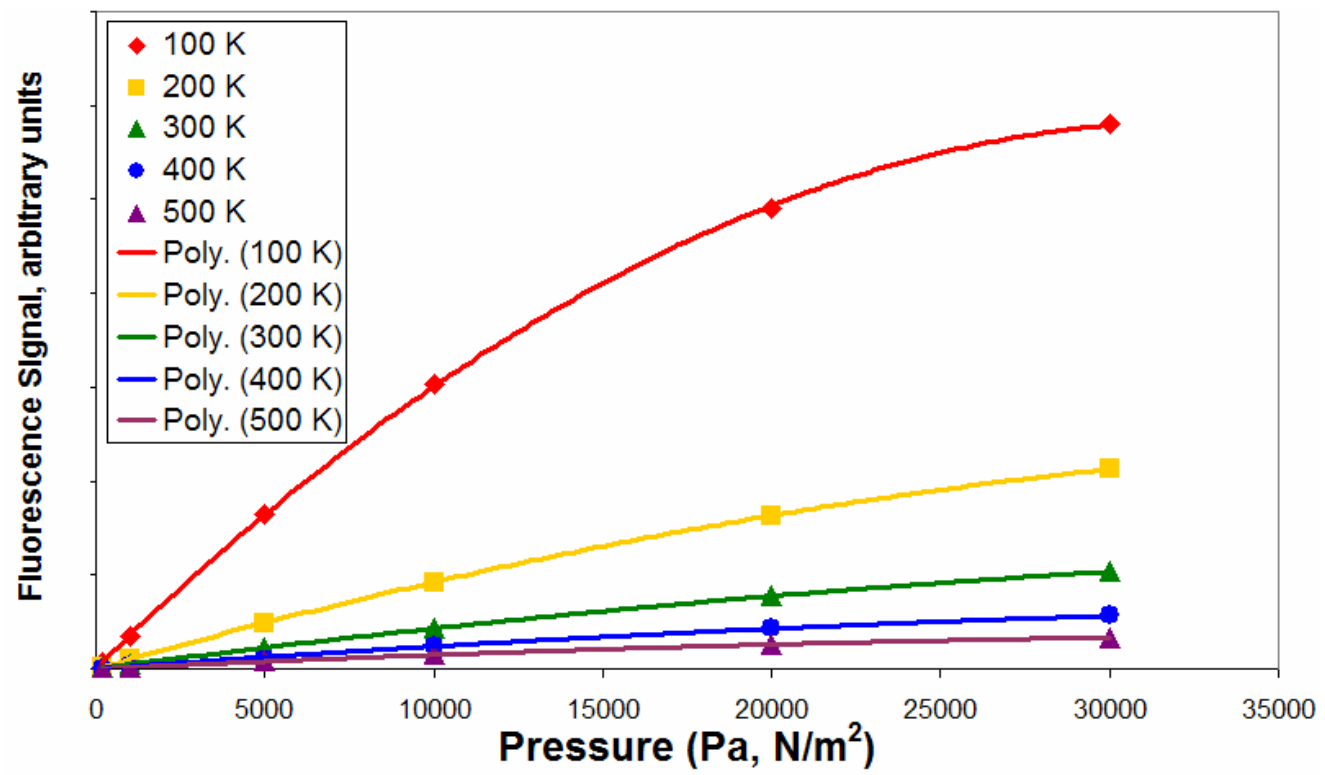

Figure 6. Dependence of fluorescence signal on temperature and pressure.

Fluorescence signal (arbitrary units) is plotted versus pressure for five temperatures covering the range anticipated in the present study.

\section{Experimental Results and Comparison with CFD}

\section{A. Jet structure comparison}

Figure 7 is a schematic showing typical flow structures exhibited by highly underexpanded jets. (The label "highly underexpanded" is used here to refer to jets having a jet pressure ratio of more than about two or three. In these highly underexpanded flows, a lens-shaped normal shock, known as a Mach disk or Riemann wave, forms. By contrast, moderately under-expanded jets - those with jet pressure ratios of less than about two-exhibit the familiar diamond-shock pattern of intersecting shocks, but lack a Mach disk.) High pressure gas exits the choked nozzle at Mach 1 and rapidly expands around the lip of the nozzle exit through expansion fans. These expansion waves reflect off the free jet boundary as compressions waves, which coalesce into a barrel shock structure. When this barrel shock structure encounters the Mach disk, an oblique reflected shock results. Additional, weakening expansion and compression waves result after this oblique shock reflects off the free jet boundary. Gas which passes through the Mach disk becomes subsonic,

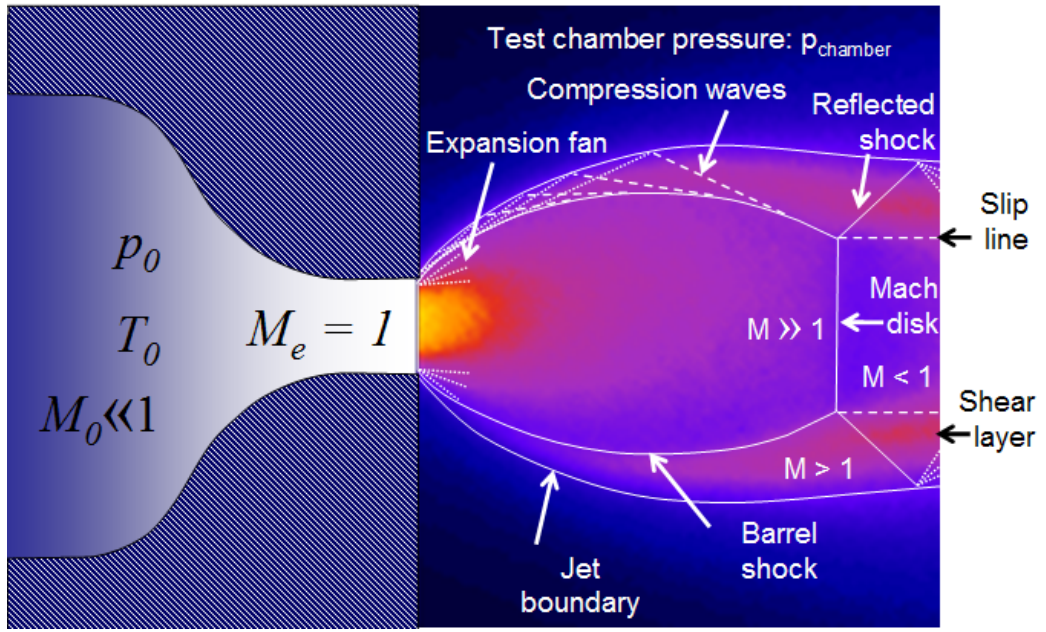

Figure 7. Highly underexpanded sonic jet flow structures. Flow structures are labeled on a false-color PLIF image of an underexpanded jet with a jet pressure ratio of approximately 30. 


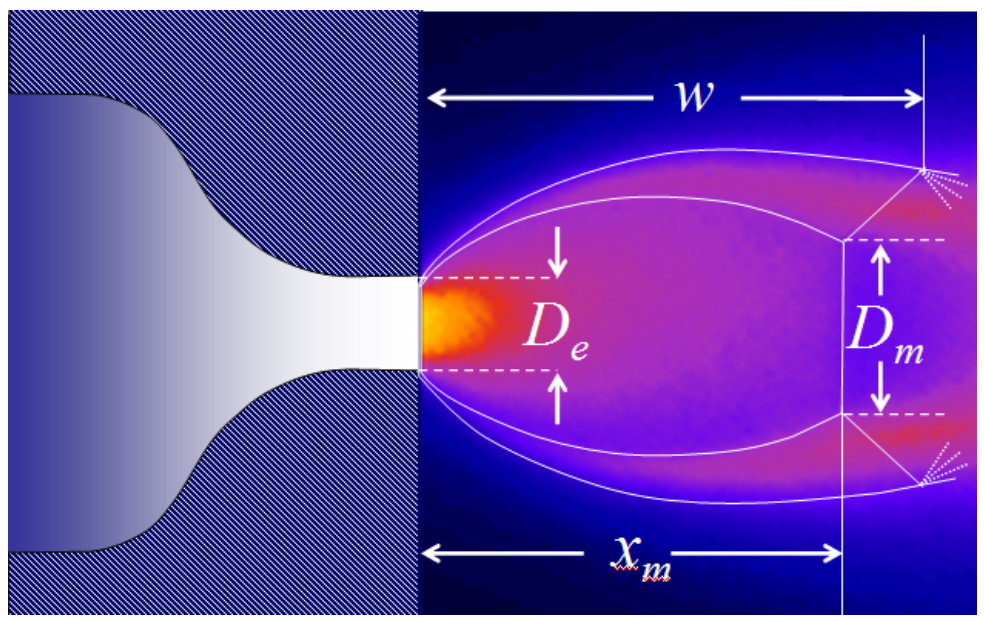

Figure 8. Flow structure dimensions. Major flow structures of interest include the following: $\boldsymbol{D}_{\boldsymbol{e}}$, the nozzle exit diameter; $\boldsymbol{D}_{\boldsymbol{m}}$, the Mach disk diameter; $\boldsymbol{x}_{\boldsymbol{m}}$, the distance to the Mach disk; and $\boldsymbol{w}$, the primary wavelength of the jet.

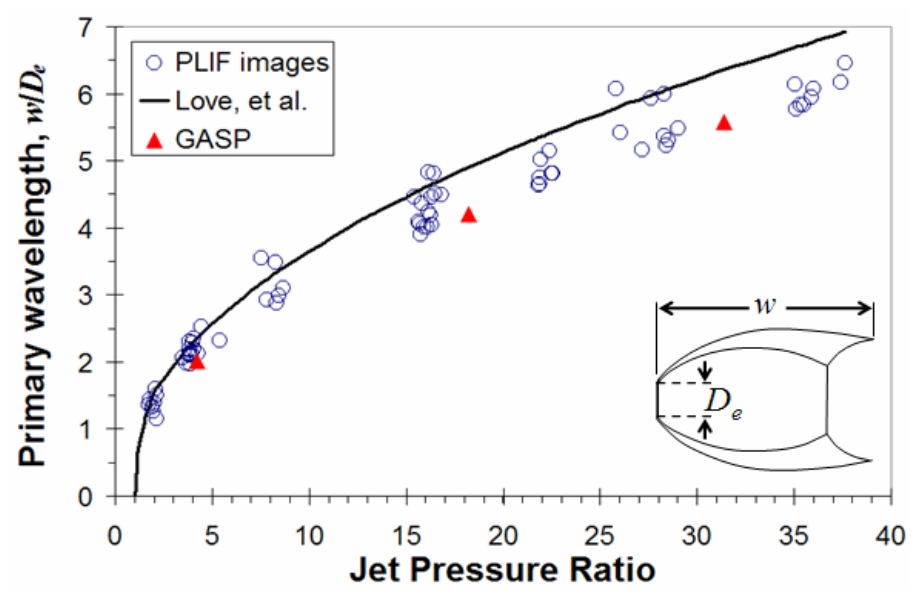

Figure 9. Jet primary wavelength. Primary jet wavelength is plotted for a range of jet pressure ratios. Data were taken at various exit Reynolds numbers.

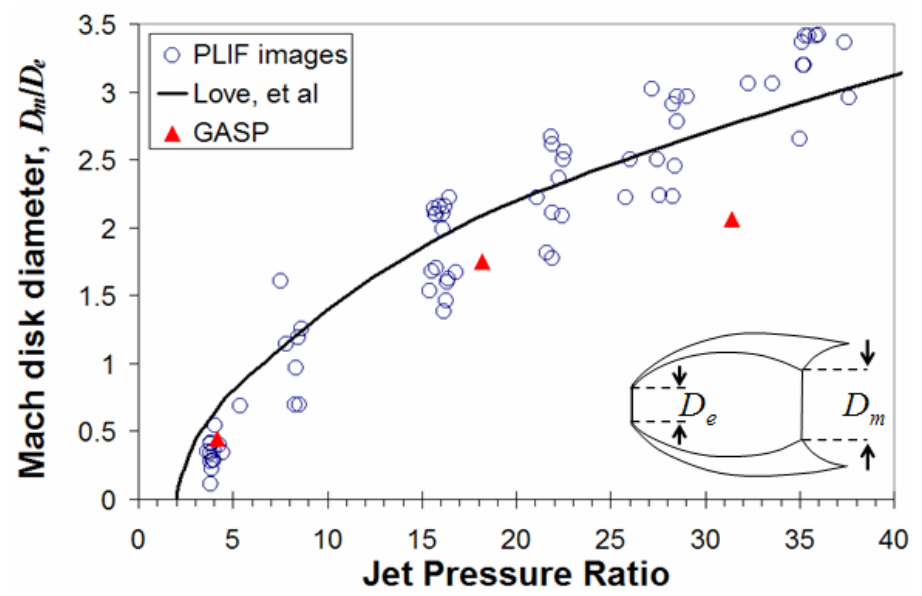

Figure 10. Mach disk diameter. Data were taken at various exit Reynolds numbers. GASP appears to underpredict this parameter at high JPR, by up to $35 \%$ for Case C. while gas which passes through the barrel shock and oblique reflected shock remains supersonic. This results in a slip line between the subsonic gas in the jet core and the supersonic gas in the shear layer along the outer edges of the jet.

Many of these flow structures, including Mach disks, barrel shocks, and shear layers, are clearly visible in the PLIF flow visualization images of this study. The location, shape, and size of such structures provide quantitative data that can be compared to CFD solutions.

For the present study, we have chosen three readily identifiable flow structure dimensions to compare with computational results. These three dimensions, indicated in Fig. 8, are the Mach disk location, $x_{m}$, the Mach disk diameter, $D_{m}$, and the primary wavelength, $w$, of the flow. To measure these dimensions from PLIF images, the magnification of the camera-lens system had to be accurately determined. Images of a scale (ruler) were obtained on nearly every day of testing. By measuring the pixel location of the hash marks on these scales and then fitting a line to these pixels locations, the spatial resolution of the imaging system was found. Flow feature locations were then recorded in terms of pixel location in the image, and these locations were then converted into a physical length. Figures 9-11 show the results of these measurements, plotted in units of nozzle diameters (as noted above, $D_{e}=0.095$ inches). In addition to experimental measurements of these quantities from approximately 100 test cases and measurements from computed results of the three CFD test cases, we have included comparisons with the seminal experimental and computational studies of Love, et al. ${ }^{2}$ In Fig. 9, it can be seen that the semi-empirical relation for primary wavelength proposed by Love et al. tends to predict a longer primary wavelength than that indicated by either our data or our GASP computations. This result was anticipated, as Love et al. cite a "tendency at the higher jet pressure ratios to overpredict the wavelength" when the nozzle exit Mach number is $l_{0}{ }^{2}$, as was the case in the present work where $M_{e}=1$. Figure 10 shows relatively good agreement between our experimental results and those of Love, et al. for the measured Mach disk diameter. It appears that GASP tends to underpredict this parameter, especially at higher jet pressure ratios. Finally, Fig. 11 shows that our experimental data tends to indicate a greater 


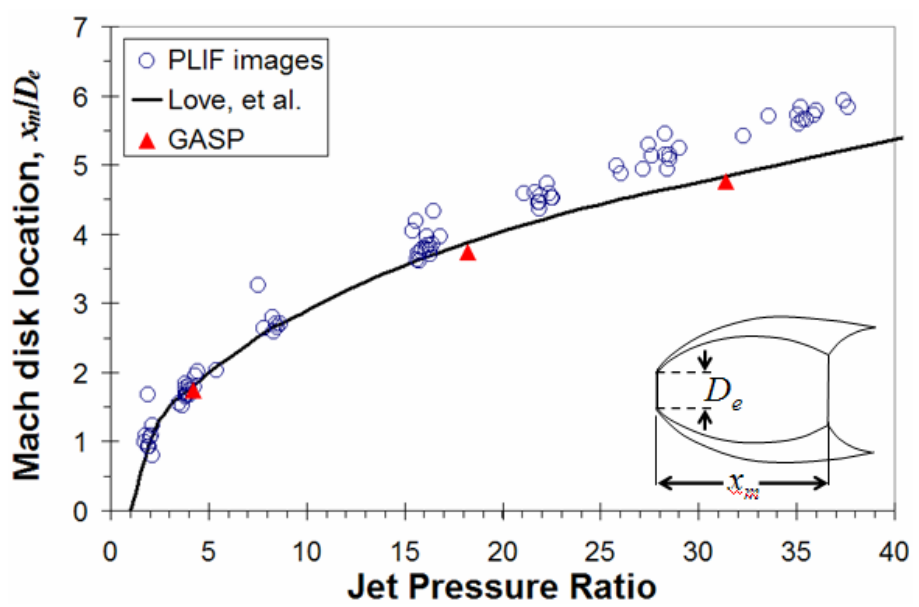

Figure 11. Mach disk location. Data were taken at various exit Reynolds numbers. While GASP solutions agree well with the results of Love, et al., they underpredict the present experimental PLIF results by approximately 15\% for Case C. distance to the Mach disk than either GASP or the experimental results of Love, et al., which show good agreement with each other.

\section{B. Qualitative image comparison}

PLIF flow visualization images taken at nearly identical conditions were processed in MATLAB ${ }^{\circledR}$ (refer to Table 1 for a list of CFD and actual test conditions). Similar grayscale color maps were applied to both the Tecplot and MATLAB ${ }^{\circledR}$ images. The results for the three test cases are shown in Figs. 12-14.

The images show relatively good qualitative agreement, although discrepancies do exist. The CFI images are brighter in regions immediately after the nozzle exit; as mentioned in the section on Computational Flow Imaging, this effect can partly be attributed to Doppler shifts of the absorption lineshape in regions where large radial components of the flow velocity are present. The PLIF images show remnants of camera artifacts, such as a slight honeycomb pattern in very bright regions of Case B and Case $\mathrm{C}$ flows. They also appear to be brighter in some regions than the CFI images, suggesting that our method of correcting for nonuniformities in the laser sheet is imperfect.

It is worth noting that the CFI formulation presented herein assumes a gas mixture consisting of $0.5 \% \mathrm{NO}$ and $99.5 \% \mathrm{~N}_{2}$ throughout. Quenching by $\mathrm{N}_{2}$ and self-quenching by $\mathrm{NO}$ has been included in the modeling, but by assuming this mixture of gases, the quenching by oxygen has been neglected. Refer again to Fig. 5, noting the regions of the flow where the NO mass fraction is less than the maximum value of $0.5 \%$. In these regions, it is expected that the experimental PLIF images will exhibit an attenuation of the fluorescence signal due to quenching by oxygen relative to the calculated signal level; this effect is evident downstream for the Case B and Case C images, whereas the Case A images have been cropped, as described previously, prior to this effect becoming wholly manifest downstream. This same effect tends to make the calculated jets appear wider than the measured jets since the $\mathrm{O}_{2}$ quenching on the jet edges is not being computed. .

The CFI GASP images are also noticeably crisper; this is because of limitations in the focus attainable by the camera lens, coupled with the spatial blurring due to collecting fluorescence from a volume with the thickness of the laser sheet $(\sim 1 \mathrm{~mm})$, and with the blurring resulting from applying a spatial filter to the data. Additionally, the fluorescence lifetime, which is on the order of 200 $\mathrm{ns}$, coupled with the relatively long camera gate width of $1 \mu \mathrm{s}$, can cause blurring in regions of high velocity in the images.

Other small discrepancies can be attributed to differences in the conditions at which the PLIF images were acquired and those that were used as input to the CFD code. Overall, the images show similar flow structures. Mach disks, barrel shocks, reflected shocks, and shear layers are all similar in size, shape, and location in the CFI and PLIF images. The similarity, in particular, of the relative intensities of these flow structures demonstrates that the modeling we have done in our computational flow imaging is a suitable approximation for these flow conditions and for our purposes in this work.

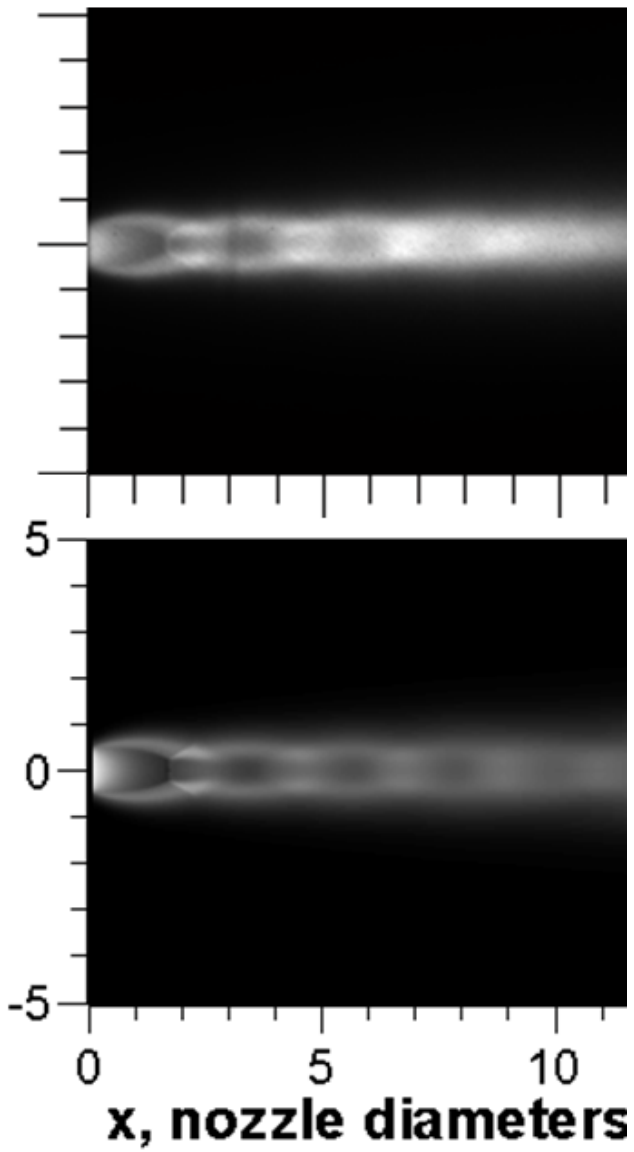

Figure 12. Case A. Qualitative comparison of PLIF flow visualization (top) and GASP CFI (bottom) for a JPR of approximately 4. 


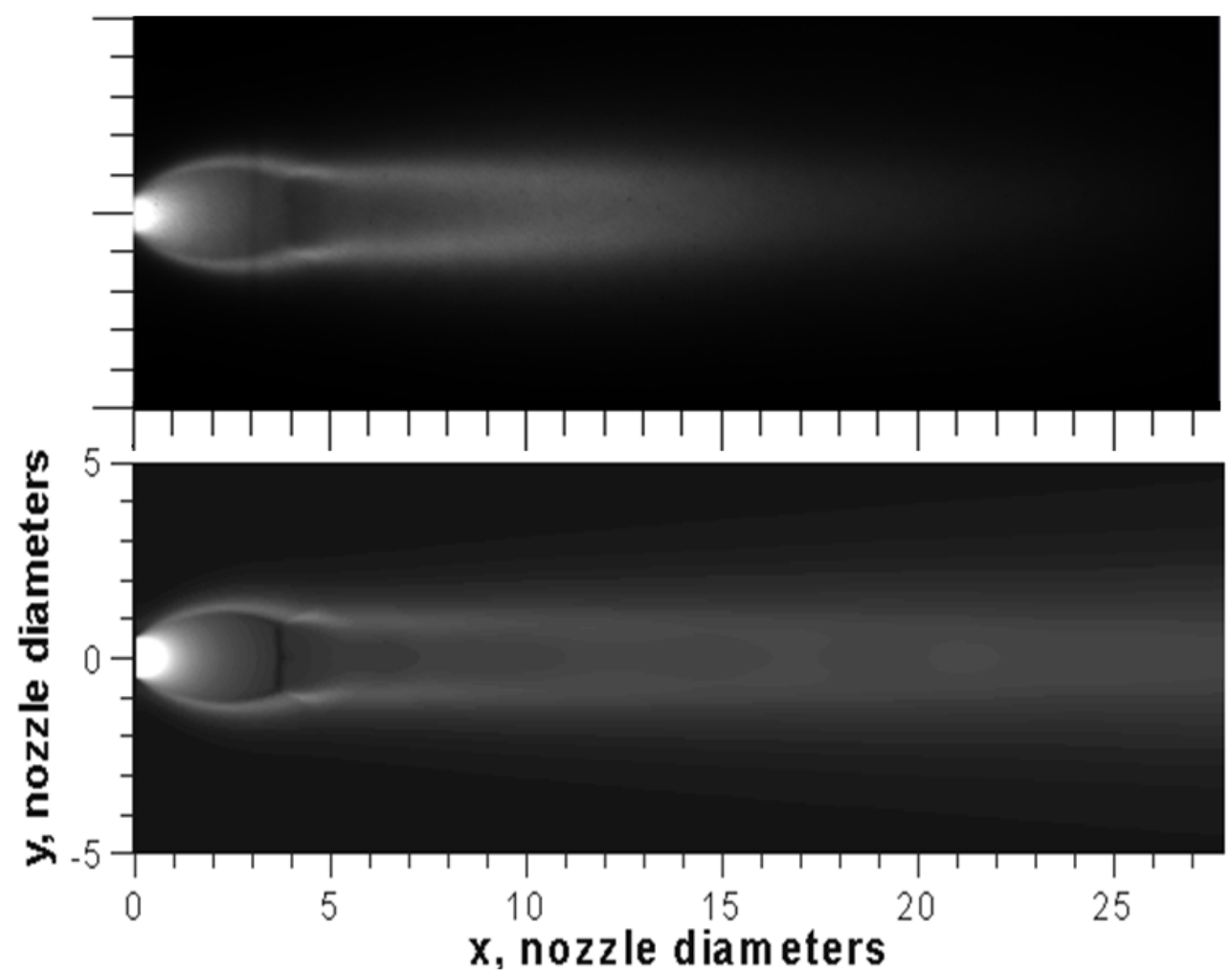

Figure 13. Case B. JPR is approximately 17 in this PLIF image (top) and 18 in this CFI image (bottom).

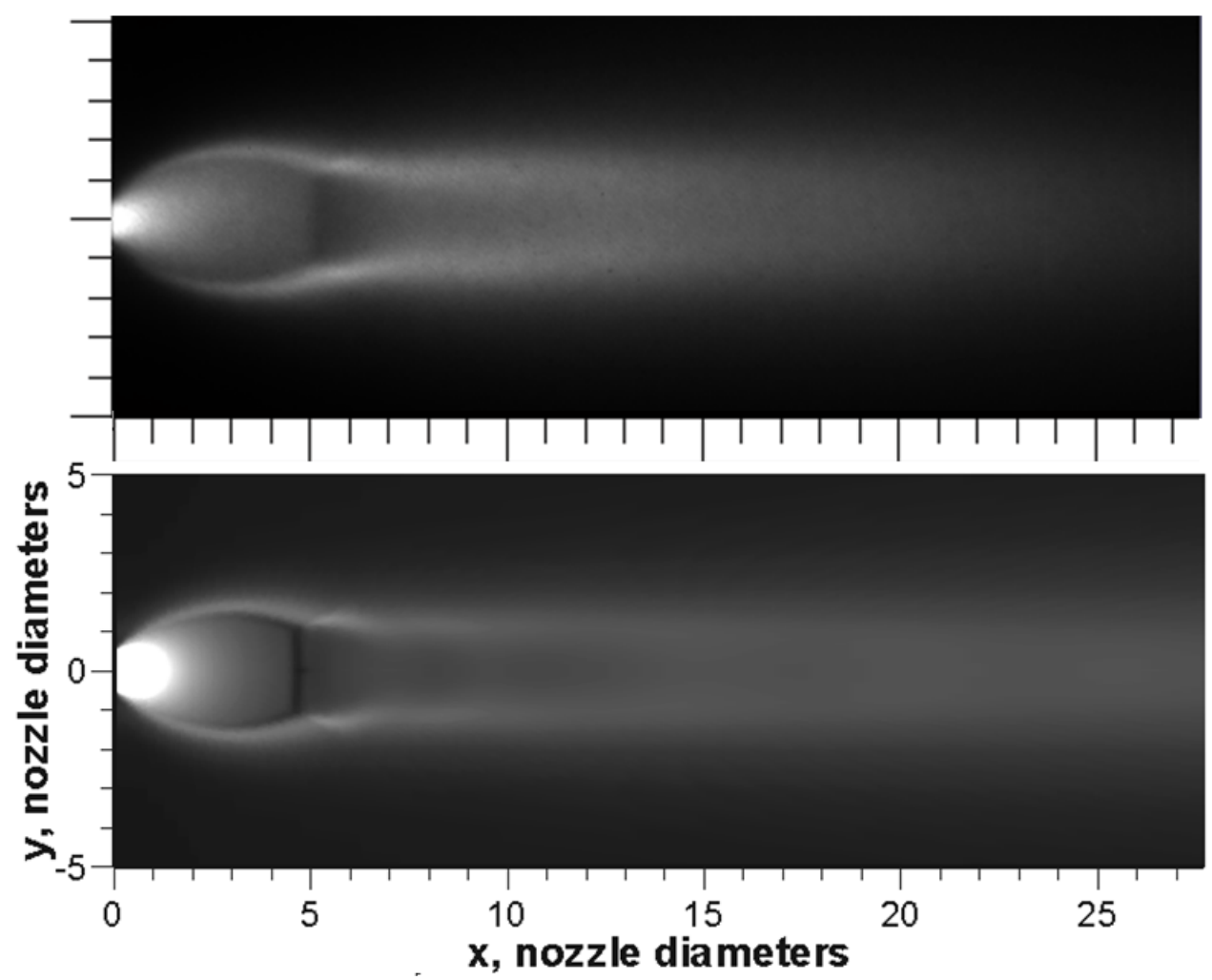

Figure 14. Case C. JPR is approximately 28 in this PLIF image (top) and 31 in this CFI image (bottom). 


\section{Conclusion}

Experimental and computational studies of highly underexpanded axisymmetric sonic free jets have been conducted as part of the shuttle return-to-flight effort at NASA Langley Research Center. These studies have endeavored to improve the understanding of the nature of such flows, as well as to compare the predictions of the CFD with experimental findings. Computation and experiment were found to be in agreement within the scatter of the experimental data for measurements of jet primary wavelength. Agreement was also found for measurements of Mach disk location and Mach disk diameter for the two cases with smaller jet pressure ratios; however, it was noted that the CFD results appear to overpredict the size of these flow features for the highest jet pressure ratio case by approximately $15 \%$ and $35 \%$, respectively. CFD calculations at a larger range of conditions will help to confirm or modify these apparent tendencies. Although not presented in this paper, 3D CFD of these test cases has been performed; further comparisons with these results are planned.

Many improvements to the measurement system are suggested, and several have been incorporated since the completion of theses tests. We have replaced the hand-pressed camera trigger with an automated camera "trigger" signal, enabling better correlation between individual PLIF images and the data acquired by the data acquisition system. We have improved our technique for characterizing the magnification and spatial resolution of our imaging system by using a two dimensional dotcard in place of a scale. From the image of this dotcard (a rectangular sheet with evenly spaced square dots in both directions), we can account for perspective effects and distortion due to the camera lens. These types of experiments would be improved if they could be conducted in a facility capable of achieving lower chamber pressures (below 1 Torr) and in which the chamber pressure could be controlled more precisely. Also, the effects of quenching by oxygen and Doppler broadening should be taken into account in the CFI PLIF calculations to draw more accurate comparisons between theory and experiment. Finally, the image processing technique described above could be improved by incorporating a separate laser profiling system, in which a second camera images the spatial profile of the beam for every laser pulse. This will be especially important in other applications where a convenient freestream region away from the main region of interest is not present.

\section{Acknowledgments}

The authors wish to acknowledge the collaborative input of Scott Halloran and Don Picetti of The Boeing Company. They also wish to thank David Alderfer, Stephen Jones, and Paul Tucker of NASA Langley Research Center for their invaluable technical assistance in running these experiments, and Chris Glass, also of NASA Langley, for performing the CFD presented in this paper. This work was funded as part of the Shuttle Return to Flight effort through Chuck Campbell of Johnson Space Center and Tom Horvath of NASA Langley Research Center.

\section{References}

${ }^{1}$ Love, E. S., Grigsby, C. E., and Lee, L. P., "Shape of Initial Portion of Boundary of Supersonic Axisymmetric Free Jets at Large Pressure Ratios,” NACA TN 4195, January 1958.

${ }^{2}$ Love, E. S., Grigsby, C. E,. Lee, L. P., and Woodling, M. J., "Experimental and theoretical studies of axisymmetric free jets," NASA TR R-6, 1959.

${ }^{3}$ Crist, S., Sherman, P. M., and Glass, D. R., "Study of the Highly Underexpanded Sonic Jet," AIAA Journal, Vol. 4, No. 1, 1966, pp. 68-71.

${ }^{4}$ C. D. Donaldson and R. S. Snedeker, "A study of free jet impingement. Part 1. Mean properties of free and impinging jets," J. Fluid Mech., Vol. 45, Part 2, pp. 281-319, 1971.

${ }^{5}$ C. D. Donaldson, R. S. Snedeker, and D. P. Margolis, "A study of free jet impingement. Part 2. Free jet turbulent structure and impingement heat transfer," J. Fluid Mech., Vol. 45, Part 3, pp. 477-512, 1971.

${ }^{6}$ Matsuda, T., Umeda, Y., Ishii, R., Yasuda, A., and Sawada, K., "Numerical and Experimental Studies on Choked Underexpanded Jets," AIAA $19^{\text {th }}$ Fluid Dynamics, Plasma Dynamics and Lasers Conference, AIAA-87-1378, Honolulu, Hawaii, 8-10 June 1987.

${ }^{7}$ Birkby, P., and Page, G. J., "Numerical Predictions of Turbulent Underexpanded Sonic Jets using a Pressure-Based Methodology," Proc. Instn. Mech. Engrs., Part G, Journal of Aerospace Engineering, Vol. 215, 2001, pp. 165-173.

${ }^{8}$ Anderson, J. D., Jr., Modern Compressible Flow: With Historical Perspective, $3^{\text {rd }}$ ed., McGraw-Hill, Boston, 2003.

${ }^{9}$ White, F. M., Viscous Fluid Flow, $2^{\text {nd }}$ ed., McGraw-Hill, Inc., New York, 1991.

${ }^{10}$ Wilkes, J. A., Danehy, P. M., and Nowak, R. J., "Fluorescence Imaging Study of Transition in Underexpanded Free Jets," Proceedings of the $21^{\text {st }}$ International Congress on Instrumentation in Aerospace Simulation Facilities [CD-ROM], Sendai, Japan, 2005, pp. 1-8.

${ }^{11}$ Wilkes, J. A., Alderfer, D. W., Jones, S. B., and Danehy, Paul M., "Portable Fluorescence imaging system for hypersonic flow facilities," JANNAF Interagency Propulsion Committee Meeting, Colorado Springs, Colorado, December 2003. 
${ }^{12}$ GASP Version 4.0 User's Manual, Aerosoft, Inc., Blacksburg, VA, 2002, ISBN 09652780-5-0.

${ }^{13}$ McDaniel, J.. C., Jr., Glass, C. E., Staack, D., and Miller, C. G., III, "Experimental and Computational Comparison of an Underexpanded Jet Flowfield," $40^{\text {th }}$ AIAA Aerospace Sciences Meeting and Exhibit, AIAA-2002-0305, Reno, NV, 14-17 January 2002.

${ }^{14}$ Bivolaru, D., Danehy, P. M., Lee, J. W., Gaffney, R. L., Jr., and Cutler, A., "Single-pulse Multi-point Multi-component Interferometric Rayleigh Scattering Velocimeter," 44 $4^{\text {th }}$ AIAA Aerospace Sciences Meeting and Exhibit, AIAA-2006-0836, Reno, NV, 09-12 January 2006.

${ }^{15}$ Ruyten, W. M., Williams, W. D., and Heltsley, F. L. , "Computational Flow Imaging for Planar Laser-Induced Fluorescence Applications (CFI-PLIF)," $18^{\text {th }}$ AIAA Aerospace Ground Testing Conference, AIAA-94-2621, Colorado Springs, CO, 20-23 June 1994.

${ }^{16}$ Danehy, P. M., Palma, P. C., Boyce, R. R., and Houwing, A. F. P., "Numerical Simulation of Laser-Induced Fluorescence Imaging In Shock-Layer Flows," AIAA Journal, Vol. 37, No. 6, 1999, pp. 715-722.

${ }^{17}$ Danehy, P. M., Palma, P. C., Houwing, A. F. P., and McIntyre, T. J., "Comparison of Theoretical Laser-Induced Fluorescence Images with Measurements Performed in a Hypersonic Shock Tunnel," $19^{\text {th }}$ AIAA Advanced Measurement and Ground Testing Technology Conference, AIAA-1996-2236, New Orleans, LA, 17-20 June 1996.

${ }^{18}$ DiRosa, M. D., "High-Resolution Line Shape Spectroscopy of Transitions in the Gamma Bands of Nitric Oxide," HGTL Report T-327, Ph.D. Dissertation, Dept. of Mechanical Engineering, Stanford Univ., Stanford, CA, May 1996.

${ }^{19}$ Chang, A. Y., Rea, E. C., Jr., and Hanson, R. K., "Temperature Dependence of Collision Broadening and Shift in the NO $\mathrm{A} \rightarrow \mathrm{X}(0,0)$ Band in the Presence of Argon and Nitrogen," Journal of Quantitative Spectroscopy and Radiative Transfer, Vol. 47, 1992, pp. 375-390.

${ }^{20}$ Eckbreth, A. C., Laser Diagnostics for Combustion Temperature and Species, $2^{\text {nd }}$ ed., Combustion Science \& Technology Book Series, Vol. 3, Taylor \& Francis, New York, NY, 1996.

${ }^{21}$ Paul, P. H., Gray, J. A., and Durant, J. L., Jr., "Collisional quenching corrections for laser-induced fluorescence measurements of NO A ${ }^{2} \Sigma^{+}$," AIAA Journal, Vol. 32, No. 8, Aug 1994, pp. 1670-1675.

${ }^{22}$ Lachney, E. R., and Clemens, N. T., "PLIF Imaging of Mean Temperature and Pressure in a Supersonic Bluff Wake," Experiments in Fluids, Vol. 24, 1998, pp. 354-363. 